\title{
A Graph-Based Method for Detecting and Classifying Clusters in Mammographic Images
}

\author{
P. Foggia ${ }^{1}$, M. Guerriero ${ }^{2}$, G. Percannella ${ }^{2}$, C. Sansone ${ }^{1}$, F. Tufano ${ }^{2}$, and M. Vento ${ }^{2}$ \\ ${ }^{1}$ Dipartimento di Informatica e Sistemistica, Università di Napoli "Federico II" \\ Via Claudio, 21 I-80125 Napoli (Italy) \\ \{foggiapa, carlosan\}@unina.it \\ ${ }^{2}$ Dipartimento di Ingegneria dell'Informazione e di Ingegneria Elettrica \\ Università di Salerno - Via P.te Don Melillo, 1 I-84084, Fisciano (SA), Italy \\ \{mguerrie, pergen, ftufano, mvento\}@unisa.it
}

\begin{abstract}
In this paper we propose a method based on a graph-theoretical cluster analysis for automatically finding and classifying clusters of microcalcifications in mammographic images, starting from the output of a microcalcification detection phase. This method does not require the user to provide either the expected number of clusters or any threshold values, often with no clear physical meaning, as other algorithms do.

The proposed approach has been tested on a standard database of 40 mammographic images and has demonstrated to be very effective, even when the detection phase gives rise to several false positives.
\end{abstract}

\section{Introduction}

Calcifications can be expression of many kinds of pathologies. Microcalcifications found in the breast hold a major diagnostic interest, because they are effective indicators of benign or malignant pathologic modifications [1]. Namely, microcalcifications found in the mammographic images represent the only symptom of neoplasiae in $30-50 \%$ of carcinomas that are not recognized in the breast [2].

Physicians agree that the presence of three or more microcalcifications grouped in a cubic centimeter of mammary tissue defines a cluster. In general, the higher the number of microcalcifications grouped in a cluster, the higher the probability that a neoplastic pathology is present. Despite the diagnostic relevance, the detection and characterization of single microcalcifications in a mammogram is often a hard task also for expert radiologists. It is not unusual that two radiologists (or even the same radiologist at different times) provide different diagnoses on the same case. This is due to the intrinsic complexity of the problem: the low injection of radiation produces a mammographic image poorly contrasted, making the microcalcifications not easily distinguishable from the mammal tissue in the background.

The availability of an automated tool for detecting clusters could allow the radiologists to concentrate their attention to suspicious areas during the review of a mammogram and to determine more reliably whether further examinations (with additional imaging tests or biopsy) are needed. This approach can improve the radiologists' diagnostic performance, in terms of sensitivity or specificity. These considerations highlight the usefulness of Computer-Aided Detection (CAD) 
techniques [3] for automatically detecting the presence of clusters in mammograms. As a consequence, in the last ten years a large interest with respect to this problem in the field of Pattern Recognition has been registered. Many authors tried to solve this problem (see $[4,5]$ for an in-depth review of the state of the art in this field) so that today some commercial tools are available on the market.

Nevertheless, most research efforts have addressed the problem of the detection of the microcalcifications; however, this aspect is only a part of the whole picture. In fact, the final goal to pursue is the detection of clusters of microcalcifications and the discrimination among benign and malignant ones, but, to the best of our knowledge, just few authors have proposed algorithms which face both these problems.

The typical approach for cluster detection first individuates the microcalcifications within the image and then aggregates them into clusters on the basis of some spatial and morphological features. The main drawback of most algorithms using this approach is that their performance is very often affected by the shape and the size of the cluster, sometimes requiring some a priori knowledge of the presumed number of clusters to be detected. Furthermore, in order to obtain an adequate cluster detection the user is often required to set some thresholds without any clear physical meaning.

In this paper, we propose a method for detecting clusters of microcalcifications which is able to overcome all the above limitations. This result has been obtained through a careful design of the algorithm for grouping the microcalcifications. In the field of Pattern Recognition there is a huge number of clustering approaches. Most algorithms aggregate the points in the feature space on the basis of the average distance of the points within the cluster. Unfortunately, this type of algorithms are not well suited to handle clusters of various shapes and sizes. A particular family of clustering algorithms are those based on graph theory. The algorithms of this family represent the clusters through undirected graphs. Each node is associated to a point in the feature space, while to each edge it is associated the distance of the connected nodes calculated in the feature space. Note that this definition of cluster does not impose any restriction with respect to the size and the shape. Furthermore, when the distance is calculated as the Euclidean distance in the image plane the method resembles the way the radiologists group the microcalcifications. For the above motivations, we decided to adopt the method described in [6], probably the most important graph based clustering method. This method requires to set only a single threshold, i.e. the maximum allowed value associated to an edge. In order to derive automatically the optimal value of this threshold, in this paper we propose an innovative method based on the use of the fuzzy c-means algorithm.

Once a cluster of microcalcifications has been detected, we also provide the information about its malignancy. The classification is carried out by a neural network on the basis of a set of features, which take into account both information computed on the whole cluster and on the single microcalcifications. We also propose and use some new features that are directly computed on the graph-based representation of the cluster.

The proposed cluster detection and classification approach has been tested on a standard database of 40 mammographic images and has shown to be very effective.

The organization of the paper is as follows: in Section 2 and Section 3 the proposed cluster detection and classification approaches are presented respectively. In Section 4 the database used is described together with the tests carried out in order to 
assess the performance of the proposed method. A comparison with the results obtained by other techniques presented in the literature is also reported. Finally, some conclusions are drawn in Section 5.

\section{Cluster Detection}

As anticipated in the introduction, a cluster is a group of at least three microcalcifications in a limited area (usually $1 \mathrm{~cm}^{2}$ ) of the mammogram. From this definition it derives that the Euclidean distance is the most important feature for clustering microcalcifications. As a consequence, the proposed algorithm first assigns the microcalcifications to candidate clusters on the basis of their relative distances; then, it eliminates clusters composed by less than three microcalcifications. It is clear that the detection of the candidate clusters constitutes the most critical phase of the whole process, especially in presence of falsely detected microcalcifications, and represents also the major innovative contribution provided by this paper.

The proposed clustering method is based on graph theoretical cluster (GTC) analysis. This family of clustering algorithms is capable of detecting clusters of various shapes, at least for the case in which they are well separated. This feature is shared only by few other clustering algorithms. This aspect is very important for the problem at hand since clusters of microcalcifications typically assume various shapes depending on the pathology and are spatially quite well separated.

In order to understand how GTC analysis is used for automatic microcalcifications clustering it is worth to review some basic terminology on the graph theory.

Graph: a graph $G$ can be defined as a set $X$ of nodes connected by a set $E$ of edges:

$$
\begin{gathered}
G=[X, E] \\
X=\left\{x_{1}, x_{2}, \ldots, x_{n}\right\} \\
E=\left\{e_{i j}=\left(x_{i}, x_{j}\right) \mid x_{i}, x_{j} \in X\right\}
\end{gathered}
$$

Path: a path $P$ of length $L$ through a graph is a sequence of connected nodes: $P=\left\langle x_{1}, x_{2}, \ldots, x_{L+1}\right\rangle$, where $\forall i \in(1, L),\left(x_{i}, x_{i+1}\right)$ is in $E$. A graph is connected if for any two nodes there is at least a path connecting them.

Cycle: a graph contains a cycle if there is a path of nonzero length through the graph $P=\left\langle x_{1}, x_{2}, \ldots, x_{l+1}\right\rangle$, such that $x_{1}=x_{l+1}$.

Spanning Tree: a spanning tree of a graph $G$ is a set of $n$ - 1 edges that connect all nodes of the graph. A tree is a connected graph $[X, T]$ with no cycles. The graph $[X, T]$ is a tree if and only if exists one and only one path between any pair of vertices.

Minimum Spanning Tree (MST): in general, it is possible to construct multiple spanning trees $\left[X, T_{i}\right]$ with $i>1$ for a graph $G$. If a weight $w(e)$ is associated with each edge $e$, then the minimum spanning tree is the set of edges forming a spanning tree such that

$$
w(\mathrm{MST})=\min _{i}\left\{\sum_{e \in T_{i}} w(e)\right\}
$$

The MST of a graph may be derived with Prim's algorithm or Kruskal's algorithm [7]. In this paper we used the Prim's algorithm. 
Forest: a graph without cycles and not connected is called a forest. Each connected component of the forest is a tree.

The proposed method starts by describing with a graph all the microcalcifications detected by an automatic algorithm: graph nodes correspond to microcalcifications, while the edges of the graph encode the spatial relationships between microcalcifications. Each microcalcification is linked by an edge to all the other ones. The weight of each edge is the Euclidean distance in the 2D space between the nodes connected by that edge. After such a graph is obtained, the GTC analysis is employed. It takes the microcalcifications as vertices in the 2D space and constructs the MST on them. By removing all the edges in the tree with weights greater than a threshold $\lambda$, we arrive at a forest containing a certain number of subtrees (clusters). In this way, the GTC method automatically groups vertices (microcalcifications) into clusters. Successively, clusters with less than three nodes are eliminated according to the above described rule.

It is worth noting that the optimal value of $\lambda$ typically depends on the specific mammogram. As a consequence, it is not possible to use a fixed value of $\lambda$ for every mammogram. Our proposal is then to determine the optimal value of $\lambda$ by reformulating the problem as the one of partitioning the whole set of edges into two clusters, according to their weights. The cluster of the edges of the MST with small weights will contain edges to be preserved, while the edges belonging to the other cluster will be removed from the MST. In order to solve this problem we employ the Fuzzy C-Means (FCM) clustering algorithm. In particular, FCM is used to separate all the edges of the MST into two clusters. Then, we remove from the MST all the edges belonging to the cluster $s$ whose center exhibits the largest value.

\section{Cluster Classification}

Cluster classification is aimed at evaluating the benignancy or malignancy of a detected cluster. In order to discriminate between benign and malignant clusters, we have defined a set of features which try to capture their differences.

Malignant clusters are usually characterized by microcalcifications with low brightness and hazy contour so that they can be easily confused with the background, while the microcalcifications of benign clusters show a high contrast with respect to the background; sometime it is possible to find regions affected by noise that are characterized by a high level of brightness. Furthermore, the typical shape of malignant clusters is elliptical, with a noticeable density of microcalcifications. For this reason, we defined a set of features which capture the brightness, density and shape characteristics of the cluster. It is worth noting that since we used a graph structure to represent the cluster the definition of some features exploits some properties of this structure. The defined features are the following:

- Brightness mean: the mean of microcalcifications brightness normalized with respect to the brightness of the area covered by the graph Brightness_mean $=\frac{m_{-} \text {micro }}{b_{-} \text {area }}$

with 


$$
m_{-} \text {micro }=\frac{\sum_{i=1}^{\text {mpix }} b m_{i}}{\text { nmicro }} \text { and } b_{-} \text {area }=\sum_{i=1}^{\text {apix }} b a_{i},
$$

where mpix is the number of microcalcification pixels in the cluster, $b m_{i}$ is the brightness of a single pixel of a microcalcification, nmicro is the number of microcalcifications in the cluster, apix is the number of pixel in the area covered by the graph and $b a_{i}$ is the brightness of a single pixel of the area.

- Brightness variance: variance of microcalcification brightness normalized with respect to the brightness of the area covered by the graph Brightness_variance $=\frac{\sum_{i=1}^{n \text { micro }}\left(b_{-} \text {micro }_{i}-m_{-} \text {micro }\right)^{2}}{\text { nmicro }} / b_{-}$area with

$$
b_{-} \text {micro }_{i}=\frac{\sum_{j=1}^{m p i x_{i}} b m_{i j}}{\text { mpix }_{i}}
$$

where mpix $_{i}$ is the number of pixels of the microcalcification $i$ and $b m_{i j}$ is the brightness of a single pixel of microcalcification $i$;

- Graph density: ratio between the number of nodes of the graph and the number of pixel of the image covered by the graph

$$
\text { Graph_density }=\frac{X^{*}}{\text { apix }}
$$

where $X^{*}$ is the number of nodes of the graph;

- Diameter/nodes ratio: ratio between the square of cluster diameter and the number of graph nodes, where the cluster diameter is the maximum distance between two graph nodes.

- Cluster compactness: mean distance among graph edges

$$
\text { Cluster_compactness }=\frac{\sum_{e \in E} w(e)}{E^{*}}
$$

where $E^{*}$ is the number of edge of graph;

- Aspect ratio: it is the ratio between the sides of the bounding box including the graph, where the bounding box is the smaller rectangle that circumscribe the graph in the image

$$
\text { Aspect_ratio }=\frac{w_{-} \text {box }}{h_{-} \text {box }}
$$

where $w \_b o x$ is the length of horizontal side expressed in pixel and $h \_b o x$ is the length of vertical side expressed in pixel.

- Graph valence: mean valence of graph nodes, where the valence of a node is the number of edges incident to the node.

Cluster classification is performed by an artificial neural network: in particular, we used a Multi Layer Perceptron (MLP) network with a hidden layer. 


\section{Experimental Results}

In order to evaluate the performance of the proposed method, tests were performed by using a standard database publicly available. It is made of 40 mammographic images, containing in the whole 105 clusters (76 malignant and 29 benign). Images were provided by courtesy of the National Expert and Training Centre for Breast Cancer Screening and the Department of Radiology at the University of Nijmegen, the Netherlands.

The proposed method assumes that microcalcifications have been already detected by a suitable method. To this aim, we chose and implemented the microcalcifications detection algorithm in [9], which uses a hierarchical pyramid neural network (HPNN) that exploits image structure at multiple resolutions for detecting clinically significant features in mammograms. Note that the latter method simply determines if a pixel of the image belongs to a microcalcification, but does not reconstruct the whole microcalcification, as required by our cluster detection and classification method. The contour of the microcalcifications is obtained by using a connected components algorithm.

\subsection{Cluster Detection}

In order to assess the performance of our method, we referred to the definitions given in [8], where a detected cluster is considered a true positive $(T P)$ if it contains two or more microcalcifications within the distance of $1 \mathrm{~cm}$, and is considered a false positive $(F P)$ if none of the microcalcifications found in the cluster are inside the ground truth circle; a false negative $(F N)$ is counted if a cluster present in the ground truth is not detected.

The performance of the proposed clustering method on the Nijmegen database was measured in terms of True Positives and False Positives per image rates and is reported in Table 1 . Note that the proposed detection method does not need any specific learning procedure as it needs only to set the parameters of the FCM algorithm. In particular, we used typical values for both the fuzziness coefficient $m=2$ and the termination criterion threshold $\varepsilon=0.05$.

The results reported in Table 1 shows that the proposed method is able to automatically reduce falsely detected clusters, yielding a very low FP per image rate.

In order to have a qualitative evaluation of the behavior of the proposed cluster detection method, in Fig. 1 are depicted the outputs of the method on two mammograms of the database. Fig. 1.a is particularly interesting since it includes clusters of different shapes and sizes. Note how the proposed system is able to correctly detect all the clusters within the image. On the other hand, in Fig. 2.b it is possible to appreciate how the system is very effective even when the microcalcification detection gives rise to several false positives.

Table 1. Performance obtained on the Nijmegen database by the proposed cluster detection method

\begin{tabular}{|c|c|}
\hline TP rate & FP per image rate \\
\hline $82.83 \%$ & $0.08 \%$ \\
\hline
\end{tabular}




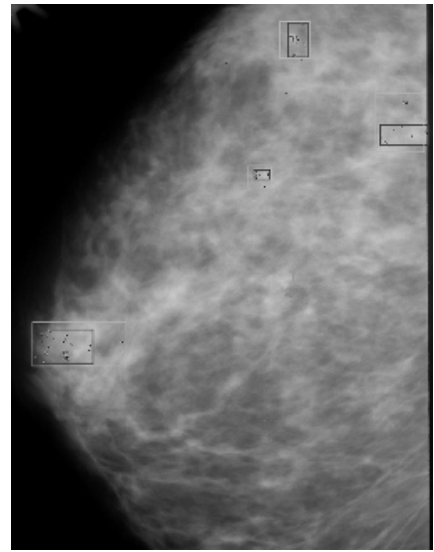

(a)

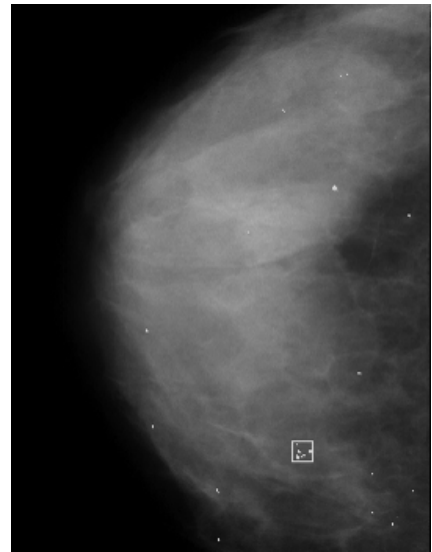

(b)

Fig. 1. (a) Light gray rectangles represent the cluster detected by the proposed method; dark rectangles account for the ground truth. (b) The white points and the white rectangle represent the microcalcification and the cluster detected by the proposed system.

\subsection{Cluster Classification}

Cluster malignancy is assessed through a neural classifier which requires an adequate training procedure. Unfortunately, the used dataset is quite small; thus, in order to have a more realistic estimate of the recognition capability of the proposed system, a $\mathrm{k}$-fold cross validation was performed. According to this experimental procedure, the whole data set is divided into $\mathrm{k}$ disjoint subsets of approximately equal size. Then the classifier is trained $\mathrm{k}$ times, each time leaving one of the subsets out of the training and then using the omitted subset to compute the recognition rate. In our case, we performed a ten-fold cross-validation; at each iteration, six parts of the database were used as a training set in each experiment, three parts as a validation set and the remaining one for testing. The validation set was used to avoid to overtrain the neural classifier. Finally, the overall performance has been calculated as the average performance over the ten iterations.

We repeated the above procedure for different number of neurons in the hidden layer of the MLP network. The best performance, reported in Table 2, was obtained when 45 neurons were used.

Data reported in table 2 show that the performance is biased toward the correct classification of the malignant clusters. This is mainly due to the fact that the composition of the dataset is unbalanced; in fact, malignant cases outnumber benign cases.

Table 2. Performance obtained on the Nijmegen database by the proposed cluster classification method; rows represent the true class, while columns the response of the classifier

\begin{tabular}{c|c|c|}
\multicolumn{1}{c}{} & \multicolumn{1}{c}{ BENIGN } & \multicolumn{1}{c}{ MALIGNANT } \\
\cline { 2 - 3 } BENIGN & $52.2 \%$ & $47.8 \%$ \\
\cline { 2 - 3 } MALIGNANT & $0 \%$ & $100 \%$ \\
\cline { 2 - 3 } & &
\end{tabular}




\subsection{Comparison with Other Methods}

We have compared our results with those reported in [8, 10-15], since in these papers the same database was used. In particular, in $[8,10-13]$ cluster detection methods are reported, while in [14] and [15] cluster classification techniques are described.

Among the above cited cluster detection methods, [10] and [8] both employ a Markov Random Field model, but in [10] a Support Vector Machine is used for reducing false positives. Methods presented in [11] and [12] are instead based on a scale-space approach; in [11] a fuzzy-based enhancement of the mammogram is also introduced as a pre-processing step. Finally, in [13] the use of wavelet coefficients together with features extracted from microcalcifications and the co-occurrence matrix is proposed.

Table 1 shows the comparison of the cluster detection results obtained by each method in terms of true positives and false positives. We have denoted our method with $G T C$, while $D E L, C H E, K A R, N E T$ and $Y U$ respectively referred to the results obtained in [10], [11], [8], [12] and [13]. From this table, it can be noted that the proposed method is outperformed by four methods in the detection of true clusters, but gives the best results in terms of false positives. The high specificity of our methods makes it particularly appealing for its use as a second-look by radiologists. Almost all the times our system detects a cluster, in fact, it is really present in the mammographic image. Therefore, a radiologist can use the detection of our system for eliminating any doubt about a particular cluster he visually found in the image without a CAD system. More in detail, the comparison with the other MRF-based methods shows that our method provides better or comparable results in terms of true positive rate and performs better in terms of false positives. Slightly worse results are obtained by our method, in terms of true positive rate, with respect to the waveletbased approach proposed in [13], while significantly better results are reached in terms of false positive. On the other hand, scale-space approaches [11, 12] perform better than our method in terms of true positives, but this is paid with a higher number of false positives. This is especially true for the method presented in [12]. Finally, it must be outlined that in [11] (where the best results in terms of true positive are reported) tests have been performed in selected areas containing all the clusters of the image, while in all our tests the whole mammografic images were used.

Table 3. Comparison of the cluster detection results obtained on the 40 images of the Nijmegen database. Best results are reported in bold.

\begin{tabular}{|l|c|c|}
\hline Method & TP rate & FP per image \\
\hline$G T C$ & $82.86 \%$ & $\mathbf{0 . 0 8}$ \\
\hline$C H E$ & $\mathbf{9 0 . 4 8 \%}$ & 0.35 \\
\hline$D E L$ & $79.05 \%$ & 0.30 \\
\hline$K A R$ & $83.81 \%$ & 1.05 \\
\hline$N E T$ & $88.57 \%$ & 0.98 \\
\hline$Y U$ & $85.71 \%$ & 0.53 \\
\hline
\end{tabular}

As regards cluster classification techniques, it is worth noting that some papers report results obtained only on a limited set of images (in [16], for example, only 18 
clusters out of 105 are considered as test set). So, we considered for comparison the methods presented in [14] and [15] whose results refer to all the 40 mammografic images of the database. In particular, in [14] a SVM using a Gaussian kernel is proposed, while MLP classifiers are employed in [15], where a multi-expert approach is also proposed in order to improve the performance of single classifiers. As regards the features for malignancy analysis, both microcalcification features and cluster features are used in [14] and [15]. Table 4 shows the comparison of the cluster classification results obtained by each method in terms of overall accuracy. We have denoted our method with Graph-based, while DES and PAP respectively referred to the results obtained in [15] and [14]. As it is evident, our method exhibits the best performance.

Table 4. Comparison of the cluster classification results obtained on the 40 images of the Nijmegen database. The best result is reported in bold.

\begin{tabular}{|l|c|}
\hline Method & Overall accuracy \\
\hline Graph-based & $\mathbf{8 4 . 2} \%$ \\
\hline$D E S$ & $75.2 \%$ \\
\hline$P A P$ & $81.0 \%$ \\
\hline
\end{tabular}

\section{Conclusions}

Mammography is a powerful tool for early diagnosis of breast cancers. A diagnosis is usually obtained by using human expertise in recognizing the presence of given patterns and types of microcalcifications. So, there are significant motivations for developing computer based support tools able to complement the radiologists work.

In this framework, we proposed a new method based on a graph-theoretical cluster analysis for automatically finding and classifying clusters of microcalcifications in mammograms. The proposed approach was tested on a standard database of mammograms and revealed to be very effective even when the microcalcification detection phase gives rise to several false positives.

\section{References}

1. M. Lanyi, Diagnosis and differential diagnosis of breast calcifications, Springer-Verlag, New York, 1986.

2. G. Coopmans De Yoldi, G. Viganotti, S. Bergonzi, C. Gerranti, G.Piragine, E. Cassano, M. Barberini, F. Rilke, U. Veronesi, "Le microcalcificazioni nei carcinomi mammari non palpabili. Analisi di 427 casi”. (in italian), Rad Med, no. 85, pp. 611-614, 1993.

3. A. Lauria, R. Palmiero, M. Imbriaco, G. Selva et al., "Analysis of radiologist performance with and without a CAD system", European Congress of Radiology, 2002.

4. H.D. Cheng, Xiaopeng Cai, Xiaowei Chen, Liming Hu, Xueling Lou, "Computer-aided detection and classification of microcalcifications in mammograms: a survey", International Journal on Pattern Recognition, Vol. 36, pp. 2967-2991, 2003.

5. K.Thangavel, M.Karnan, R.Sivakumar, A. Kaja Mohideen, "Automatic Detection of Microcalcification in Mammograms - A Review", International Journal on Graphics, Vision and Image Processing, Vol. 5, pp. 31-61, 2005. 
6. C.T. Zahn, "Graph-theoretical methods for detecting and describing gestalt clusters", IEEE Transactions on Computers, Vol. 20(1), pp. 68-86, January 1971.

7. E. Horowitz, S. Sahni, Fundamentals of Computer Algorithms, Computer Science Press, 1978.

8. N. Karssemeijer, "Adaptive Noise Equalization and Recognition of Microcalcification Clusters in Mammograms", Int. Journal of Patt. Rec. and Artificial Intelligence, Vol. 7, no. 6, pp. 1357-1376, 1993.

9. P. Sajda, C. Spence, J. Pearson, "Learning contextual relationships in mammograms using a hierarchical pyramid neural network", IEEE Transactions on Medical Imaging, Vol. 21, No. 3, pp. 239-250, 2002.

10. C. D'Elia, C. Marrocco, M. Molinara, G. Poggi, G. Scarpa, F. Tortorella, "Detection of Microcalcifications Clusters in Mammograms through TS-MRF Segmentation and SVMbased Classification”. IEEE International Conference on Pattern Recognition, Vol. 3, pp. 742-745, 2004.

11. T. Netsch and H. Peitgen, "Scale-Space Signatures for the Detection of Clustered Microcalcifications in Digital Mammograms", IEEE Trans. on Medical Imaging, Vol. 18, no. 9, pp. 774-786, 1999.

12. H.D. Cheng, J. Wang and X. Shi, "Microcalcification Detection Using Fuzzy Logic and Scale Space Approach”, Pattern Recognition, Vol. 37, pp. 363-375, 2004.

13. S. Yu, L. Guan, "A CAD system for the automatic detection of clustered microcalcifications in digitized mammogram films", IEEE Transactions on Medical Imaging, Vol. 19, no. 2, pp. 115-126, 2000.

14. A. Papadopoulos, D.I. Fotiadis, A. Likas, "Characterization of clustered microcalcifications in digitized mammograms using neural networks and support vector machines", Artificial Intelligence in Medicine, 2006 (in press).

15. M. De Santo, M. Molinara, F. Tortorella, M. Vento, "Automatic classification of clustered microcalcifications by a multiple expert system", Pattern Recognition, Vol. 36, pp. 14671477, 2003.

16. B. Verma, J. Zakos, "A computer-aided diagnosis system for digital mammograms based on fuzzy-neural and feature extraction techniques", IEEE Transactions on Inform. Technol. Biomed., vol 5, no. 1, pp. 46-54, 2001. 